\title{
A CONSTRUCTION OF UNCOUNTABLY MANY WEAK VON NEUMANN TRANSFORMATIONS
}

BY

\author{
KARL DAVID 1
}

\begin{abstract}
We define weak von Neumann transformations and discuss some of their properties, using several examples of countable classes of these transformations. Then we construct an uncountable class by the cutting-and-stacking method. We show that each member of this class is ergodic and has zero entropy.
\end{abstract}

1. Introduction. Let $(X, \mathscr{B}, m)$ be the closed-open unit interval $X=[0,1)$, with $\mathscr{B}$ the Borel $\sigma$-field and $m$ Lebesgue measure. We denote by $G(X)$ the group of all one-to-one, measurable and nonsingular transformations of $X$ onto itself, and let $\mathbf{N}$ be the set of positive integers.

DEFINITION 1.1. A measure-preserving transformation $T \in G(X)$ is called a weak von Neumann transformation if, for each $n \in \mathbf{N}$, there exists a measurable partition $\mathscr{D}_{n}(T)=\left\{D_{1}^{n}, D_{2}^{n}, \ldots, D_{2^{n}}^{n}\right\}$ of $X$ satisfying

(i) $D_{i}^{n}=T^{i-1}\left(D_{1}^{n}\right)$ and

(ii) $D_{i}^{n}=D_{i}^{n+1} \cup D_{i+2^{n}}^{n+1}$

for $1<i \leqslant 2^{n}$.

If in addition we have

(iii) the $\sigma$-field generated by $\left\{D \in \mathscr{D}_{n}(T) \mid n \in \mathbf{N}\right\}$ equals $\mathscr{B}(\bmod 0)$, we say that $T$ is a von Neumann transformation.

It is well known that any two von Neumann transformations are isomorphic. The standard realization of such a transformation is

ExAmple 1.1. For each $n \in \mathbf{N}$, let $e_{n}=\left\{C_{1}^{n}, C_{2}^{n}, \ldots, C_{2^{n}}^{n}\right\}$ consist of the dyadic closed-open subintervals of $X$ of rank $n$, ordered from left to right. A transformation $T: X \rightarrow X$ is defined by finding for any $x \in X$ the interval $C_{i}^{n}$ of minimum rank containing $x$ for which $i \neq 2^{n}$, and setting $T(x)=x+3 / 2^{n-1}$. This defines a piecewise-linear measure-preserving transformation in $G(X)$ which satisfies the definition of a von Neumann transformation. Note that it linearly maps $[0,1 / 2)$ to $[1 / 2,1),[1 / 2,3 / 4)$ to $[1 / 4,1 / 2)$ and in general

$$
\left[\left(2^{n-1}-1\right) / 2^{n-1},\left(2^{n}-1\right) / 2^{n}\right) \text { to }\left[1 / 2^{n}, 1 / 2^{n-1}\right) \text {, }
$$

and thus is best depicted in cutting-and-stacking terms (see Example 6.4 in [1, p. 82]). When one considers its action on points of $X$ in their binary expansion, it becomes clear why it is often referred to as the "adding machine" transformation. We however shall refer to it as the von Neumann-Kakutani transformation, or simply as the $V N K$ map.

Received by the editors August 7, 1978.

AMS (MOS) subject classifications (1970). Primary 28A65.

${ }^{1}$ This paper is excerpted from the author's doctoral dissertation. 0002-9947/80/0000-0056/\$04.50 
It is not difficult to show that the VNK map is ergodic and has zero entropy. When we consider certain of its powers, we obtain examples of strictly weak von Neumann transformations (again, weak VNK maps, for short).

EXAMPLE 1.2. Let $T$ be the VNK map. Then the transformation $T^{2}$ is a weak VNK map which is not ergodic; its nontrivial invariant sets are in fact $[0,1 / 2)$ and $[1 / 2,1)$. It acts on each of these sets independently the way $T$ acts on $X$. In general, each $T^{2^{n}}$ for $n \in N$ is a nonergodic weak VNK map whose invariant sets are the dyadic intervals of rank $n$ (and their unions), acting on each of these intervals independently the way $T$ does on $X$. As powers of $T$, they all still have zero entropy.

A countable class of ergodic weak VNK maps can also be constructed. One way is to take the independent cutting-and-stacking transformations discussed by Friedman in [1, p. 89ff], and for which entropy computations are given by Shields [2], whose terminology we follow here. Specifically, given $n \in \mathbf{N}$, by taking initial configurations of uniform height 2 with $2^{n}$ columns, we obtain an ergodic weak VNK map with entropy $\frac{1}{2} \log \left(2^{n}\right)$. A simple modification of the cutting-and-stacking pattern of Example 1.2, one which sufficiently "mixes" the columns at each stage, should also result in a countable class of ergodic weak VNK maps, but this time all of zero entropy. Thus countable classes of weak VNK maps with varying properties are easily obtainable. The purpose of this paper is to show by explicit construction that in fact an uncountable class exists. It is a remarkable fact that the removal of only condition (iii) from Definition 1.1 should lead from essentially only one possible example to uncountably many.

\section{The construction.}

Definition 2.1. Let $\Omega$ be the set of all infinite sequences of zeros and ones. For each $\omega \in \Omega$, a weak VNK map $T_{\omega} \in G(X)$ is defined inductively as follows. Suppose $\omega_{n}, n \in \mathbf{N}$, is the $n$th entry of $\omega$. For the first stage, we define the action of $T_{\omega}$ via linear translation by

$$
[0,1 / 2) \rightarrow[1 / 2,1) \text { if } \omega_{1}=0
$$

and

$$
[0,1 / 4) \rightarrow[3 / 4,1) \quad[2 / 4,3 / 4) \rightarrow[1 / 4,2 / 4) \quad \text { if } \omega_{1}=1
$$

Thus the configuration for $T_{\omega}$ of height two has one column if $\omega_{1}=0$ and two columns if $\omega_{1}=1$.

For the second stage, we continue the stacking procedure via the linear translations:

$$
\begin{gathered}
{[2 / 4,3 / 4) \rightarrow[1 / 4,2 / 4) \text { if } \omega_{1} \omega_{2}=00,} \\
{[6 / 8,7 / 8) \rightarrow[5 / 8,6 / 8) \quad[2 / 8,3 / 8) \rightarrow[1 / 8,2 / 8) \text { if } \omega_{1} \omega_{2}=10,} \\
{[8 / 16,9 / 16) \rightarrow[7 / 16,8 / 16) \quad[14 / 16,15 / 16) \rightarrow[1 / 16,2 / 16)} \\
{[10 / 16,11 / 16) \rightarrow[5 / 16,6 / 16) \quad[12 / 16,13 / 16) \rightarrow[3 / 16,4 / 16)} \\
\text { if } \omega_{1} \omega_{2}=01,
\end{gathered}
$$




$$
\begin{array}{rlrl}
{[24 / 32,25 / 32) \rightarrow[23 / 32,24 / 32)} & {[14 / 32,15 / 32) \rightarrow[1 / 32,2 / 32)} \\
{[26 / 32,27 / 32) \rightarrow[21 / 32,22 / 32)} & {[12 / 32,13 / 32) \rightarrow[3 / 32,4 / 32)} \\
{[28 / 32,29 / 32) \rightarrow[19 / 32,20 / 32)} & {[10 / 32,11 / 32) \rightarrow[5 / 32,6 / 32)} \\
{[30 / 32,31 / 32) \rightarrow[17 / 32,18 / 32)} & {[8 / 32,9 / 32) \rightarrow[7 / 32,8 / 32)} \\
\text { if } \omega_{1} \omega_{2}=11 .
\end{array}
$$

The columns of the stacking diagrams of height four should be drawn from left to right in the same order as presented by the formulas above. Note that the four possible configurations at this stage have differing powers of two (namely $2^{0}, 2^{1}, 2^{2}$ and $2^{3}$ ) as their number of columns.

In general, suppose a configuration of height $2^{n-1}$ is given; that is, we know the first $n-1$ entries of $\omega$. If $\omega_{n}=0$, the configuration of height $2^{n}$ shall have the same number of columns as the preceding one. This is done by cutting each column at hand in half, going from the left half of the first to the right half of the last to form the first column of the new diagram, the left half of the last to the right half of the first to form the second column, etc., continuing to alternate directions until finally the left half of the first column in the right half of the diagram goes to the right half of the last column in the left half of the diagram.

If, however, $\omega_{n}=1$, we wish the number of columns to increase by a factor of $2^{2^{n-1}}$, and do this by cutting each existing column into $2 \cdot 2^{2^{n-1}}$ equal-sized pieces and stacking the subcolumns in the same order as above, starting with the extreme left and right subcolumns and alternating the direction.

We note in passing that $T_{\omega}$ for the zero-sequence is just the VNK map itself; all the others are strictly weak VNK maps. The definition insures that if $\omega \neq \tilde{\omega}$, then from some point on (namely from that corresponding to the first entry where $\omega$ and $\tilde{\omega}$ differ), the diagrams for $T_{\omega}$ and $T_{\tilde{\omega}}$ never have the same number of columns. Ir fact, one will always have at least twice as many columns as the other, although the lead can alternate according to which of $\omega$ or $\tilde{\omega}$ has a higher frequency of ones up to the given point, and where in the sequence they occur. As an example, if $\omega=1110 \ldots$, then the number of columns in the diagram for $T_{\omega}$ of height sixteen is $2^{1} \cdot 2^{2} \cdot 2^{4} \cdot 2^{0}=128$; whereas if $\tilde{\omega}=0001 \ldots$, the corresponding diagram for $T_{\tilde{\omega}}$ has $2^{0} \cdot 2^{0} \cdot 2^{0} \cdot 2^{8}=256$ columns.

\section{Ergodicity.}

TheOREM 3.1. For any $\omega \in \Omega, T_{\omega}$ is ergodic.

Proof. If $\omega$ is the zero-sequence, then $T_{\omega}$ is ergodic. Otherwise, suppose $J$ is an interval in some column at a stage of the construction where the configuration for $T_{\omega}$ has height $2^{n}$ and $2^{m}$ columns for some $n, m \in \mathbf{N}$. We show first that $m\left(J^{*}\right)=$ 1 , where $J^{*}=\bigcup_{k=-\infty}^{\infty} T^{k} J$.

Now $J^{*}$ includes the entire column containing $J$, and consequently $m\left(J^{*}\right) \geqslant$ $1 / 2^{m}$. Since in the construction subcolumns of a given column always go to subcolumns of a different column, we find at the next stage that the proportion of columns containing a subinterval of $J$ has doubled, independently of $\omega_{n+1}$, and 
hence $m\left(J^{*}\right) \geqslant 1 / 2^{m-1}$. The columns of the diagram of height $2^{n+1}$ which contain subintervals of $J$ are all adjacent, forming a block of length 2 if $\omega_{n+1}=0$ or of length $2 \cdot 2^{2^{n}}$ if $\omega_{n+1}=1$. Moreover, the preceding and succeeding blocks can also be grouped in this way. Hence at the next stage, again no subcolumn containing a subinterval of $J$ goes to a subcolumn also containing a subinterval of $J$, and so $m\left(J^{*}\right)>1 / 2^{m-2}$ (assuming $m \geqslant 2$ ). In a finite number of steps, we reach a point where either the left or the right half of the diagram for $T_{\omega}$ has a subinterval of $J$ in each column; thus at the next stage every column will have one, so that $m\left(J^{*}\right)=1$.

Now suppose $A$ is an arbitrary set of positive measure, and let $\varepsilon>0$ be given. By the Lebesgue density theorem, there is an interval $J$ from some stage of the construction such that $m(A \cap J) \geqslant(1-\varepsilon) m(J)$.

Write $J=\cup{ }_{i=1}^{2^{n}} J_{i}$ (disj.) for some $n \in \mathbf{N}$, where each $J_{i}$ is a subinterval of $J$, one per each column of the diagram for $T_{\omega}$ at some later stage. Suppose the column heights at that stage are $2^{m}, m \in \mathbf{N}$. We compute

$$
\begin{aligned}
m\left(A^{*}\right) & \geqslant m\left[(A \cap J)^{*}\right] \geqslant m\left[\bigcup_{i=1}^{2^{n}} \bigcup_{k=-2^{m}}^{2^{m}} T_{\omega}^{k}\left(A \cap J_{i}\right)\right] \geqslant \sum_{i=1}^{2^{n}} 2^{m} m\left(A \cap J_{i}\right) \\
& =2^{m} m(A \cap J) \geqslant 2^{m}(1-\varepsilon) m(J)=(1-\varepsilon) m\left(J^{*}\right)=1-\varepsilon .
\end{aligned}
$$

Since $\varepsilon$ is arbitrary, $m\left(A^{*}\right)=1$, completing the proof.

4. Nonisomorphism. In this section we select an uncountable subset $\Omega^{\prime}$ of $\Omega$ such that if $\omega, \tilde{\omega} \in \Omega^{\prime}$ are distinct, then $T_{\omega}$ and $T_{\tilde{\omega}}$ are not isomorphic.

LEMMA 4.1. Let $T_{1}$ and $T_{2}$ be isomorphic ergodic weak VNK maps. Let $\left\{C_{1}, C_{2}, \ldots, C_{2^{n}}\right\}$ and $\left\{D_{1}, D_{2}, \ldots, D_{2^{n}}\right\}$ be the partition sets of $X$ determined by the diagrams of height $2^{n}$ for $T_{1}$ and $T_{2}$ respectively (we do not assume however that $C_{i} \leftrightarrow D_{i}$ for each $i$ under the isomorphism). Suppose $m(A)>0$ and $A \subseteq C_{i}$ for some $i$, and that $A \leftrightarrow B$ under the isomorphism. Then $B \subseteq D_{j}(\bmod 0)$ for some $j$.

Proof. Suppose not, i.e. $B=B_{1} \cup B_{2}(\bmod 0)$, where $B_{1} \subseteq D_{j}$ and $B_{2} \subseteq D_{k}$ for $j \neq k$, with $m\left(B_{1}\right), m\left(B_{2}\right)>0$. Since $T_{2}$ is ergodic, there exists an integer $m$ such that $m\left(T_{2}^{m} B_{1} \cap B_{2}\right)>0$. Let $A_{1} \leftrightarrow B_{1}$ and $A_{2} \leftrightarrow B_{2}$, so that $A=A_{1} \cup A_{2}(\bmod 0)$. But then $m\left(T_{1}^{m} A_{1} \cap A_{2}\right)=0$, and since $T_{1}^{m} A_{1} \leftrightarrow T_{2}^{m} B_{1}$, this is a contradiction.

We note in passing that this lemma is not true in the nonergodic case.

LEMMA 4.2. Let $T$ and $S$ be measure-preserving transformations in $G(X)$, isomorphic via a map $\phi$ such that $\phi T=S \phi$ a.e. Let $A \in \mathscr{B}$ and $\varepsilon>0$ be given, with $D \in \mathscr{B}$ satisfying $m(D \triangle \phi A)<\varepsilon / 2$. Then for every $n \in \mathbf{N}$,

$$
\left|m\left(T^{n} A \cap A\right)-m\left(S^{n} D \cap D\right)\right|<\varepsilon .
$$

Proof. Since $\left(S^{n} D \cap D\right) \triangle\left(S^{n} \phi A \cap \phi A\right) \subseteq S^{n}(D \triangle \phi A) \cup(D \triangle \phi A)$, we have

$$
m\left[\left(S^{n} D \cap D\right) \triangle\left(S^{n} \phi A \cap \phi A\right)\right]<\varepsilon .
$$


Thus

$$
\begin{aligned}
\left|m\left(T^{n} A \cap A\right)-m\left(S^{n} D \cap D\right)\right| \leqslant & \left|m\left(T^{n} A \cap A\right)-m\left(S^{n} \phi A \cap \phi A\right)\right| \\
& +\left|m\left(S^{n} \phi A \cap \phi A\right)-m\left(S^{n} D \cap D\right)\right| \\
& =\left|m\left(S^{n} \phi A \cap \phi A\right)-m\left(S^{n} D \cap D\right)\right| \\
& \leqslant m\left[\left(S^{n} D \cap D\right) \triangle\left(S^{n} \phi A \cap \phi A\right)\right]<\varepsilon .
\end{aligned}
$$

LEMMA 4.3. Under the same hypotheses as above, suppose that $T$ and $S$ are in addition ergodic weak VNK maps, and that $A$ is contained on a level at some stage of the construction for $T$. Consider any subsequent stage where $A$ meets $n$ levels. Then there exists $A^{\prime} \subseteq A$ which is the intersection of $A$ with some level $\gamma$ at this stage and for which $m\left(D^{\prime} \triangle \phi A^{\prime}\right)<\varepsilon / 2 n$, where $D^{\prime} \subseteq D$ is the intersection of $D$ with the level $\phi(\gamma)$ at the corresponding stage of the construction for $S$.

Proof. Let $\gamma_{i}, 1 \leqslant i \leqslant n$, be the levels that meet $A$ at the stage of $T$ under consideration, with $A_{i}=A \cap \gamma_{i}$, so that $A=\cup{ }_{i=1}^{n} A_{i}$. Then we may write $D=\cup{ }_{i=1}^{n} D_{i} \cup \tilde{D}$, where $D_{i}=D \cap \phi \gamma_{i}$ and $\tilde{D}$ is the part of $D$ not contained in the $n$ levels $\phi \gamma_{i}$. Then $(\bmod 0)$,

$$
D \triangle \phi A=\bigcup_{i=1}^{n}\left(D_{i} \Delta \phi A_{i}\right) \cup \tilde{D}(\mathrm{disj}),
$$

which follows from Lemma 3.1.

Now suppose that for all $i, m\left(D_{i} \triangle \phi A_{i}\right)>\varepsilon / 2 n$. Then

$$
m(D \triangle \phi A)=\sum_{i=1}^{n} m\left(D_{i} \triangle \phi A_{i}\right)+m(\tilde{D}) \geqslant \varepsilon / 2
$$

a contradiction, and the result follows.

Next we will need an algorithm to enable us to follow a piece (i.e. one of the dyadic intervals that comprise a level) of a given diagram as it breaks up during succeeding stages.

Definition 4.1. Suppose $\omega \in \Omega$ is not the zero-sequence, and consider a configuration for $T_{\omega}$ with $2^{n}$ columns, $n \in \mathbf{N}$. An initial code is a consecutive numbering of the pieces, from left to right, of a given level of the configuration, i.e. the row array

$$
12 \cdots 2^{n-1} 2^{n-1}+1 \cdots 2^{n}-12^{n} \text {. }
$$

Initial codes are continued for each subsequent stage of the transformation as follows. At the next stage, the given level splits into two levels, so that the new code will have two rows. We wish the digit in a given place of either of these rows to indicate from which coded piece of the previous stage the piece which this place represents is taken. The bottom row of the new code will stand for the pieces in the lower of the two levels in question, the top row for the higher level. Thus the code 
for the next stage will be

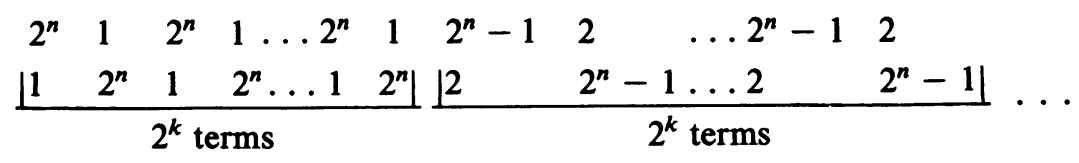

$$
\begin{aligned}
& 2^{n-1}+1 \quad 2^{n-1} \quad \ldots 2^{n-1}+12^{n-1} \\
& \frac{2^{n-1} \quad 2^{n-1}+1 \ldots 2^{n-1} \quad 2^{n-1}+1 \mid}{2^{k} \text { terms }}
\end{aligned}
$$

for some $k \in \mathbf{N}$, where $k=1$ if and only if the appropriate term of $\omega$ is zero.

As the code is continued through subsequent stages in the manner described, the length increases each time a one is encountered in $\omega$, and the height doubles each time.

The following are the formal algorithms for code continuations.

Lemma 4.4. Consider a code of length $2^{n}$ with $2^{p}$ rows. The next code is constructed by the use of the following two formulas. We wish to determine where the digit in position $j$ of row $q$ of the given code ends up in the new code. Let $m$ be either zero or the appropriate power of two that determines by what factor $2^{m}$ the number of columns in the configuration increases in passing from the stage corresponding to the given code to the next. The change of position formulas for $j$ are

(1) $j \rightarrow 2^{n+1} j-k$ in row $q$ of the new code for $1<j<2^{n-1}$,

$j \rightarrow 2^{m+1}\left(2^{n}-j\right)+(k+1)$ in row $q$ of the new code for $2^{n-1}+1<j<2^{n}$, where $k=1,3, \ldots 2^{m+1}-3,2^{m+1}-1$;

(2) $j \rightarrow 2^{m+1} j-k+1$ in row $2^{p}+q$ of the new code for $1<j<2^{n-1}$,

$j \rightarrow 2^{m+1}\left(2^{n}-j\right)+k$ in row $2^{p}+q$ of the new code for $2^{n-1}+1<j<2^{n}$, again for $k=1,3, \ldots, 2^{m+1}-3,2^{m+1}-1$.

Proof. These formulas are the algebraic equivalent of the original verbal description of the construction.

We refer to formula (1) as the "first, last algorithm" or " $f, l$ rule," since the new positions of the digits on these rows are determined by taking the first and last digits (repeating if necessary), then the second and penultimate, etc., digits from the old rows. Similarly, formula (2) becomes the "last, first algorithm" or " $l, f$ rule." Observe that if $m \neq 0$, the construction forces $m>n+1$. In any event the new code length is $2^{n+m}$.

Definition 4.2. Consider an arbitrary row of a code of length $2^{n}$. For $1<j<$ $2^{n-1}$, we say that digits in positions $j$ and $2^{n}+1-j$ are in antipathetic position, while digits in positions $j$ and $2^{n-1}+j$ are in sympathetic position.

Given an integer $i, 1<i<n$, suppose we partition the row into $2^{n-i}$ consecutive blocks of length $2^{i}$ each. Then a pair of digits within any one of the blocks is said to be in (n-i)-weakly sympathetic position if the digits are "relatively sympathetic," i.e. in sympathetic position within that block (thus the notions of 0 -weak sympathy and sympathy coincide). 
EXAMPLE 4.1. For the initial code 12345678, the antipathetic pairs are

$$
(1,8) \quad(2,7) \quad(3,6) \quad(4,5) \text {, }
$$

the sympathetic pairs are

$$
(1,5) \quad(2,6) \quad(3,7) \quad(4,8)
$$

the 1-weakly sympathetic pairs are

$$
(1,3) \quad(2,4) \quad(5,7) \quad(6,8)
$$

and the 2-weakly sympathetic pairs are

$$
(1,2) \quad(3,4) \quad(5,6) \quad(7,8) \text {. }
$$

LEMMA 4.5. Let $n \geqslant 2$ and consider row $q$ of a code of length $2^{n}$ with $2^{p}$ rows, with $m$ such that the next code has length $2^{n+m}$. Then

(1) for $1 \leqslant i \leqslant n-1$, an ( $n-i)$-weakly sympathetic pair of digits becomes $2^{m}(n-i-1)$-weakly sympathetic pairs of digits on each of rows $q$ and $2^{p}+q$ of the next code, while

(2) a sympathetic pair forms $2^{m}$ antipathetic pairs on each of these rows and

(3) if $m=0$, each antipathetic pair becomes an (n-1)-weakly sympathetic pair, whereas if $m \neq 0$, the new weakly sympathetic pairs of orders $n-1, n, \ldots, n+m-2$ are all of the form $(j, j), 2^{m-1}$ such pairs of each order for each digit $j$ occurring in the given code, while $2^{m}$ new $(n+m-1)$-weakly sympathetic pairs are again determined by each old antipathetic pair.

Proof. These facts follow from the algorithms for code continuations (Lemma 4.4), and verifying them for a specific example is more illuminating than following the technical proof. Thus for the code in Example 4.1 (where $n=3, p=0$ ), if $m=0$, the new code is

$$
81726354 \quad 18273645,
$$

whereas if, say, $m=4$ it is

$$
\begin{array}{cccc}
8181 \ldots 81 & 7272 \ldots 72 & 6363 \ldots 63 & 5454 \ldots 54 \\
|1818 \ldots 18| & |2727 \ldots 27| & \mid 3636 \ldots 36 & |4545 \ldots 45| \\
\mid 2^{5} \text { terms } & 2^{5} \text { terms } & 2^{5} \text { terms } & 2^{5} \text { terms } \mid \\
2^{2} \text { groups of terms }
\end{array}
$$

LEMMA 4.6. Any code satisfies the property that if two columns begin with the same digit, then corresponding places of the two have the same digit. That is, columns that begin with the same digit are identical.

Proof. Assume $n>2$ and $m$ are as in Lemma 4.5 (the case $n=1$ we discuss at the end). We observe that the columns of a given code that combine to form the columns of the next code are those in antipathetic position. For, by the $f, l$ rule for $1<j<2^{n-1}$ and the $l, f$ rule for $2^{n-1}+1<2^{n}+1-j<2^{n}$, we have $j \rightarrow 2^{m+1} j-k$ 
terms after the bar. Since $\omega_{m+1}=1$ necessarily, we have

$$
\gamma_{m+1}=B B \ldots B|B| \frac{R R \ldots R R \ldots R}{2^{m} \text { terms }} B
$$

and

$$
\tilde{\gamma}_{m}=B B \ldots B \mid \frac{R R R \ldots R}{2^{m-1} \text { terms }} B,
$$

with $m \geqslant 2$, thus verifying the lemma for the first relevant stage.

Denoting the length of a truncated end sequence $\gamma_{n}$ by $l\left(\gamma_{n}\right)$, the induction hypothesis breaks down into (even/odd) cases. Suppose first that the lemma holds for all safe terms up to and including those determined by the index $2 n$. Assuming $l\left(\tilde{\gamma}_{2 n}\right)>l\left(\gamma_{2 n}\right)$, we bound the maximum difference in lengths by examining the case where $\omega_{2 m}=0$ and $\tilde{\omega}_{2 m}=1$ for all $m \leqslant n$. We then have

$$
l\left(\tilde{\gamma}_{2 n}\right)=\left(\sum_{j=0}^{2 n-1} 2^{j}\right)+2 n \text { and } l\left(\gamma_{2 n}\right)=\left(\sum_{j=0}^{n-1} 2^{2 j}\right)+2 n,
$$

so that the difference is

$$
\sum_{j=0}^{n-1} 2^{2 j+1}<2^{2 n}-1
$$

Setting $l\left(\gamma_{2 n}\right)=a$ and $l\left(\tilde{\gamma}_{2 n}\right)=\tilde{a}$, we have $2<\tilde{a}-a<2^{2 n}-1$. The lemma is to be verified for the new occurrences of a $B$ at the ends of $\gamma_{2 n+1}$ and $\tilde{\gamma}_{2 n+1}$. In the first case, the $B$ is in position $a+2^{2 n}+1$; comparing with position $\tilde{a}+2^{2 n}+1$ for the new occurrence of a $B$ in $\tilde{\gamma}_{2 n+1}$, a match would force $a=\tilde{a}$ and thus is impossible. In the second case, we have a $B$ in position $\tilde{a}+2^{2 n}+1$. The next two possibilities for a $B$ in $\gamma$ are the terms

$$
a+2^{2 n}+2 \quad\left(\text { if } \omega_{2 n+2}=0\right)
$$

and

$$
a+2^{2 n}+2^{2 n+1}+2 \quad\left(\text { if } \omega_{2 n+2}=1\right) .
$$

But $a+2^{2 n}+2=\tilde{a}+2^{2 n}+1 \Rightarrow \tilde{a}-a=1$, while $a+2^{2 n}+2^{2 n+1}+2=\tilde{a}+2^{2 n}$ $+1 \Rightarrow \tilde{a}-a=2^{2 n+1}+1$. Both of these are impossible, and no further possibilities need be checked, completing the induction step for even indices. The odd case is handled similarly and is omitted.

We now state and prove the main result.

THEOREM 4.1. The transformations $T=T_{\omega}$ and $\tilde{T}=T_{\tilde{\omega}}$ are not isomorphic.

Proof. Suppose the transformations were isomorphic via an isomorphism $\phi$, such that $\phi T=\tilde{T} \phi$ a.e. Set $A^{\prime}=[0,1 / 4)$, one of the intervals comprising a piece of the (identical) configurations of height two for both $T$ and $\tilde{T}$. Let $\varepsilon^{\prime}=\frac{1}{8} m\left(A^{\prime}\right)=\frac{1}{32}$. Let $D^{\prime}$ be a finite union of intervals such that $m\left(D^{\prime} \triangle \phi A^{\prime}\right)<\varepsilon^{\prime} / 2$. Without loss of generality we assume the intervals comprising $D^{\prime}$ to be dyadic and all of the same rank. Then there is some configuration for $\tilde{T}$ (say that determined by $\tilde{\omega}_{m}$ ) which $D^{\prime}$ exactly "fits," i.e. each interval in $D^{\prime}$ is a piece (or union of pieces) of that configuration. In the latter case, we can simply break $D^{\prime}$ down further to force an 
exact fit. Note that $A^{\prime}$ will meet $2^{m-1}$ levels of the diagram at this same stage for $T$. By Lemma 4.3, there will be a level $\gamma$ at this stage for $T$ such that, if $A=A^{\prime} \cap \gamma$, $D=D^{\prime} \cap \phi \gamma$, and $\varepsilon=\varepsilon^{\prime} / 2^{m-1}$, then $m(D \triangle \phi A)<\varepsilon / 2$.

Note that at this stage $D$, as well as $A$, consists entirely of pieces on only one level, by Lemma 4.1. Also, since $m(A)=1 / 2^{m-1} m\left(A^{\prime}\right)$, the relationship $\varepsilon=\frac{1}{8} m(A)$ is preserved. By Lemma 4.2 , the proof will be complete if we can produce an integer $n$ such that

$$
\left|m\left(T^{n} A \cap A\right)-m\left(\tilde{T}^{n} D \cap D\right)\right|>\varepsilon .
$$

For $T$, we work with the code continuations of the initial code $t^{\prime}=12$ representing the base level of the diagram of height two, so that 1's correspond to pieces taken from $A^{\prime}$ in the continuations. When we reach the stage determined by the given $m$, we take from the code continuation the row corresponding to $A$ and assume without loss of generality that it begins with a 1 , so that when we continue this new initial code $t$ (which we do not renumber, i.e. we retain 1's and 2's as they are), we are assured that a 2 returns to the end of the base row infinitely often (Remark 4.1).

Thus suppose $n>m$ is such that the base row of the code continuation $t_{n}$ of $t$ corresponding to the diagram of height $2^{n}$ has a 2 on the end. By Lemma 4.5, all antipathetic pairs on all rows of $t_{n}$ are of the form $(1,2)$. Consequently, in forming $t_{n+1}$, we stack over each column of $t_{n}$ another column of $t_{n}$ with the property that the 1's and 2's are interchanged. Hence $m\left(T^{2^{n}} A \cap A\right)<\frac{1}{2} m(A)$, since half the l's in $t_{n+1}$ are in the top half of that code and are "missed" by the 1's in the bottom half when applying $T^{2^{n}}$.

If in addition it happens that $t_{n+1}$ has a 1 on the end of its base row, then all antipathetic pairs are either $(1,1)$ or $(2,2)$, and so by looking at a typical column of $t_{n+2}$ we see that $m\left(T^{2^{n}} A \cap A\right) \leqslant \frac{1}{4} m(A)$.

Turning our attention to $\tilde{T}$, we let $\tilde{t}^{\prime}=12$ be as above, with $\tilde{t}$ analogous to $t$ there (replacing $A$ by $D$ ). The situation is quite different from that for $T$, however-we have no assurance of a $1 \leftrightarrow D$ (or $2 \leftrightarrow D$ ) correspondence. Hence this time we do renumber $\tilde{t}$, say with the integers $j$ for $1 \leqslant j \leqslant 2^{k}$, for some $k$. If we let $J=\left\{1,2, \ldots, 2^{k}\right\}$, then we have a subset $J^{\prime}$ of $J$ associated with $D$, defined by letting $j \in J^{\prime}$ if the $j$ th piece of the level of the configuration corresponding to $\tilde{t}$ is from $D$. Again, $J^{\prime}$ does not necessarily contain half the indices in $J$. However, it is not empty, since then $D \triangle \phi A=\phi A$, contradicting $m(D \triangle \phi A)<\frac{1}{2} \varepsilon=\frac{1}{16} m(A)$.

We consider a stage, say that for a configuration of height $2^{n}$ ( $n$ not necessarily the same as above), where all antipathetic pairs in $\tilde{t}_{n}$ are of the form $(j, j), j \in J$. By the definition of $\Omega^{\prime}$ and Lemma 4.5, this happens infinitely often. Lemma 4.6 yields, by considering $\tilde{t}_{n+1}$,

$$
m\left(\tilde{T}^{2^{n}} D \cap D\right) \geqslant \frac{1}{2} m(D) .
$$

As before, we consider an additional possibility, namely when $\tilde{t}_{n+1}$ again has antipathetic pairs $(j, j), j \in J$. Then

$$
m\left(\tilde{T}^{2^{n}} D \cap D\right)>\frac{3}{4} m(D) .
$$


and

$$
\left(2^{n}+1-j\right) \rightarrow 2^{m+1}\left[2^{n}-\left(2^{n}+1-j\right)\right]+k^{\prime}=2^{m+1} j-\left(2^{m+1}-k^{\prime}\right),
$$

with a one-to-one correspondence $k \leftrightarrow 2^{m+1}-k^{\prime}$. The calculations for $2^{n-1}+1<j$ $<2^{n}$ are similar.

Now, a given code can be traced back to an initial code, which has the (obvious) property that weakly sympathetic pairs are unique, i.e. if $\left(j, j^{\prime}\right)$ and $\left(j, j^{\prime \prime}\right)$ are weakly sympathetic pairs of the same order, then $j^{\prime}=j^{\prime \prime}$ (similarly for antipathetic pairs). Lemma 4.5 shows that this property continues to hold for all code continuations; moreover, the pairs will be the same on all rows. Thus, if two columns of some code are identical, the antipathetic columns are also identical, so that an induction argument proves the lemma (for $n \geqslant 2$ ).

REMARK 4.1. We treat the case $n=1$ separately because here we wish to make some additional observations on code continuations. The initial code is 12 , so that the notions of antipathy and sympathy coincide. Hence Lemma 4.5(1) cannot apply, at least until the code has increased in length, if it ever does. The rest of that lemma remains valid and therefore, of couse, so does Lemma 4.6. The observations we wish to make (whose proofs are trivial) are

(1) the base row of any code always begins with the digit 1 ,

(2) a 2 returns to the end of the base row infinitely often,

(3) if at any time we choose a row beginning wth a 1 from a code to form a new initial code, then properties (1) and (2) hold for that code continuation; if the row we choose begins with a 2 , they hold with the roles of the digits interchanged.

Definition 4.3. Let $\Omega^{\prime}=\left\{\omega \in \Omega \mid \omega_{n}=1\right.$ for all odd $\left.n\right\}$, an uncountable subset of $\Omega$.

Given distinct $\omega, \tilde{\omega} \in \Omega^{\prime}$, we shall show that $T_{\omega}$ and $T_{\tilde{\omega}}$ are not isomorphic. First we need a technical lemma to allow us to compare codes for $T_{\omega}$ and $T_{\tilde{\omega}}$.

DEFINITION 4.4. Let a sequence of codes for a transformation $T_{\omega}, \omega \in \Omega^{\prime}$, be given. An end sequence for this sequence is formed by taking the digits in the last position of the base row of each of the codes in succession. Thus if the initial code has length $2^{n}$, the associated end sequence is just an infinite sequence, each term of which is an integer $j, 1 \leqslant j \leqslant 2^{n}$.

EXAMPLE 4.2. For the initial code 12345678 presented before, the associated end sequence will begin $8532 \ldots$

However, the next term will depend on the occurrence of a zero or a one at the appropriate place in the expansion of $\omega$ : specifically, that place which determines whether or not the initial code increases in length at the next stage. In the first case, the next term is again an 8, but in the second, there will be $m$ l's followed by a single $8, m$ being some power of two, as Lemma 4.5 shows. Clearly, an end sequence for a $T_{\omega}$ with $\omega \in \Omega^{\prime}$ will contain arbitrarily long strings of l's.

Preparatory to stating the final lemma, suppose we have initial codes $t_{1}$ and $\tilde{t}_{1}$ for $T=T_{\omega}$ and $\tilde{T}=T_{\tilde{\omega}}, \omega, \tilde{\omega}^{\prime} \in \Omega^{\prime}$, taken from the first stage of each of these transformations. Since $\omega_{1}=\tilde{\omega}_{1}=1$, we have $t_{1}=12$ and $\tilde{t}_{1}=12$. Thus the associated end sequences $\gamma$ and $\tilde{\gamma}$ begin $\gamma=22 \ldots$ and $\tilde{\gamma}=22 \ldots$ 
The second stage will determine more terms of the end sequences. Say $\omega_{2}=0$. Then the continuation of $t_{1}$ is

$$
t_{2}=\frac{21}{12}
$$

and so we have $\gamma=222 \ldots$

Suppose however that $\tilde{\omega}_{2}=1$. We then have

$$
\tilde{t}_{2}=\begin{aligned}
& 21212121 \\
& 12121212
\end{aligned}
$$

so that $\tilde{\gamma}=22112 \ldots$ Our convention in this stage-by-stage determination of the end sequences is not to anticipate required ones in the expansions of $\omega$ and $\tilde{\omega}$.

Suppose we consider the configurations for $T$ and $\tilde{T}$ at some stage (say where the diagrams have height $2^{m}$ ) where the number of columns already differs, and look at the parts of $\gamma$ and $\tilde{\gamma}$ that can be determined up to and including that time. Call these truncated sequences $\gamma_{m}$ and $\tilde{\gamma}_{m}$. We ask, by how little can the lengths of $\gamma_{m}$ and $\tilde{\gamma}_{m}$ differ? The example, where we computed $\gamma_{2}$ and $\tilde{\gamma}_{2}$, already gives the answer, namely by 2 . For here we have made $\omega$ and $\tilde{\omega}$ differ in the earliest possible entry, the second. Easy computations, based on the fact that $\omega_{m}$ determines 1 term in the end sequence if $\omega_{m}=0$ and $2^{m-1}+1$ terms if $\omega_{m}=1$, show that if the first difference in $\omega$ and $\tilde{\omega}$ comes later, i.e. $m \geqslant 4$, then the difference in length between $\gamma_{m}$ and $\tilde{\gamma}_{m}$ is greater. Moreover, the difference persists (or increases) at all subsequent stages.

Definition 4.5. Let $\omega, \tilde{\omega} \in \Omega^{\prime}$ be distinct. A term 1 in the end sequences $\gamma$ and $\tilde{\gamma}$ for the initial codes $t_{1}$ and $\tilde{t}_{1}$ is called mandatory if it is determined by $\omega_{n}$ or $\tilde{\omega}_{n}$ for $n$ odd. We relabel each such 1 with an $R$. All other terms in the end sequences are called optional and each is relabelled with a $B$. Finally, a term in $\gamma$ or $\tilde{\gamma}$ is called safe if it is determined at a stage where $T$ and $\tilde{T}$ differ in column size.

For the preceding example, all terms of the end sequences after the second are safe, and the relabelling scheme gives us

$$
\gamma_{2}=B B B \text { and } \tilde{\gamma}_{2}=B B R R B
$$

with the requirement $\omega_{3}=\tilde{\omega}_{3}=1$ yielding

$$
\gamma_{3}=B B B R R R R B \text { and } \tilde{\gamma}_{3}=B B R R B R R R R B
$$

LEMMA 4.7. Let (relabelled) end sequences $\gamma$ and $\tilde{\gamma}$ be as above. Then corresponding safe terms cannot both be labelled $B$.

Proof. The first safe terms are those determined by the first $m$ for which $\omega_{m} \neq \tilde{\omega}_{m}$. We then have

$$
\gamma_{m}=B B \ldots B \mid B
$$

and

$$
\tilde{\gamma}_{m}=B B \ldots B \mid \frac{R R \ldots R}{2^{m-1} \text { terms }} B
$$

or vice versa. We use the vertical bars to separate previously determined terms from those determined at this stage. The lemma is obviously true for the first $2^{m-1}$ 
Now, Lemma 4.7 was proven for a situation where both initial codes were 12 . It remains valid here, where we have renumbered one of the codes, since mandatory 1's (which were relabelled by $R$ 's) in the end sequences are the same whether we renumber or not. Hence we apply this lemma to the end sequences $\gamma$ and $\tilde{\gamma}$ (labelled with $R$ 's and $B$ 's) corresponding to $t$ and $\tilde{t}$ respectively, and find a safe position for which the term of $\gamma$ is a $B$ which was originally a 2 . The corresponding term of $\tilde{\gamma}$ must thus be an $R$. The final step in the proof now breaks down into two cases, depending on the next term of $\gamma$.

Suppose first that the term is an $R$. Then for the appropriate $n$,

$$
m\left(T^{n} A \cap A\right)<\frac{1}{4} m(A)=2 \varepsilon,
$$

while

$$
m\left(\tilde{T}^{n} D \cap D\right) \geqslant \frac{1}{2} m(D) .
$$

Recalling that $m(D \triangle \phi A)<\varepsilon / 2$, we estimate

$$
m(D)>m(D \cap \phi A)=m(D \cup \phi A)-m(D \triangle \phi A)>m(A)-\varepsilon / 2 .
$$

Thus

$$
m\left(\tilde{T}^{n} D \cap D\right)-m\left(T^{n} A \cap A\right) \geqslant \frac{1}{2} m(D)-\frac{1}{4} m(A) \geqslant \frac{1}{4} m(A)-\frac{1}{4} \varepsilon=\frac{7}{4} \varepsilon,
$$

and so $\left|m\left(T^{n} A \cap A\right)-m\left(\tilde{T}^{n} D \cap D\right)\right| \geqslant \varepsilon$, in violation of Lemma 4.2.

In the other case, we suppose the next term of $\gamma$ is a $B$, so that the corresponding term of $\tilde{\gamma}$ must be an $R$. Then

$$
m\left(T^{n} A \cap A\right)<\frac{1}{2} m(A)=4 \varepsilon,
$$

while

$$
m\left(\tilde{T}^{n} D \cap D\right) \geq \frac{3}{4} m(D) .
$$

We obtain $m\left(\tilde{T}^{n} D \cap D\right)-m\left(T^{n} A \cap A\right) \geqslant \frac{13}{8} \varepsilon$, once again violating Lemma 4.2. This concludes the proof.

\section{Entropy.}

Definition 5.1. Let $T \in G(X)$ and suppose $Q \subseteq \mathscr{B}$ is a sub- $\sigma$-field invariant under $T$, i.e. $T^{-1}(A) \in \mathbb{Q}$ for all $A \in \mathbb{Q}$. Then $T_{\mathscr{Q}}$ the (point) transformation $T$ on the space $\left(X, \mathcal{Q}, m_{\mathscr{Q}}\right)$, is called a factor transformation of $T$. If $\mathscr{Q}=\{\varnothing, X\}(\bmod 0)$, the factor is trivial.

EXAMPLE 5.1. Given $n \in \mathbf{N}$, let $\mathbb{Q}$ consist of the dyadic intervals of rank $n$ and their unions, and let $T$ be the VNK map. Then $Q$ is invariant under $T$ and so $T_{\mathscr{Q}}$ is defined. If we identify all points within each dyadic interval in $\mathcal{Q}$, then $T_{\mathscr{Q}}$ is essentially a rotation on $2^{n}$ points of equal mass.

We shall determine the entropies of the transformations $T_{\omega}$ for $\omega \in \Omega^{\prime}$ indirectly, using a theorem of Sinai [3]. Let $\mathcal{H}(T)$ denote the entropy of any measure-preserving $T \in G(X)$. The theorem implies that if $\mathcal{H}(T)>0$ for an ergodic $T$ and $0<\mathcal{H}<\mathcal{H}(T)$, then $T$ has a (nontrivial) factor $T_{\mathscr{Q}}$ with $\mathcal{H}\left(T_{\mathscr{Q}}\right)=\mathcal{H}$. Moreover, $T_{\mathscr{Q}}$ is mixing. Thus if $\mathcal{H}\left(T_{\omega}\right)>0$, then $T_{\omega}$ would have to have a nontrivial mixing factor. We show this is impossible, and hence $\mathcal{H}\left(T_{\omega}\right)=0$. 
Theorem 5.1. Let $\omega \in \Omega^{\prime}$ and set $T=T_{\omega}$. Then $\mathcal{H}(T)=0$.

Proof. Let $E \in \mathscr{B}, 0<m(E)<1$, be arbitrary. If we can show that

$$
\limsup _{n \rightarrow \infty} m\left(T^{n} E \cap E\right)>m^{2}(E)
$$

then $E$ cannot satisfy the mixing condition

$$
m\left(T^{n} E \cap E\right) \rightarrow m^{2}(E),
$$

and so $T$ cannot have any nontrivial mixing factors.

Suppose $A$ is a finite union of dyadic intervals of the same rank. Then $A$ fits some configuration for $T$ exactly. If $\gamma$ is a level of that configuration meeting $A$, we can code it and identify the pieces that belong to $A$. Whenever we encounter a string of $m$ mandatory l's in the associated end sequence, then arguing as in the proof of Theorem 4.1, we will have, for the appropriate $n$,

$$
m\left[T^{n}(A \cap \gamma) \cap(A \cap \gamma)\right] \geqslant\left(1-1 / 2^{m}\right) m(A \cap \gamma)
$$

Applying this argument to all such levels we obtain

$$
m\left(T^{n} A \cap A\right) \geqslant\left(1-1 / 2^{m}\right) m(A) .
$$

In fact, we can find a sequence $\left\{n_{k}\right\}, k \in \mathbf{N}$, such that

$$
m\left(T^{n_{k}} A \cap A\right) \geqslant\left(1-1 / 2^{k}\right) m(A) \text {. }
$$

Let $\varepsilon>0$ be such that $m(E)-3 \varepsilon>m^{2}(E)$, and find a finite union $A$ of dyadic intervals of the same rank such that $m(E \triangle A)<\varepsilon / 8$. Finally, find $k$ such that $1 / 2^{k} m(A)<\varepsilon / 2$, and set $n=n_{k}$. Then

$$
\begin{aligned}
& m\left[T^{n}(E \cap A)\right.\cap(E \cap A)] \\
& \leqslant m\left(T^{n} E \cap E\right) \leqslant m\left[T^{n}(E \cap A) \cap(E \cap A)\right]+3 \varepsilon / 8
\end{aligned}
$$

and

$$
m\left[T^{n} A \cap(X \backslash A)\right]=m\left(T^{n} A\right)-m\left(T^{n} A \cap A\right)<m(A)-\left(1-1 / 2^{k}\right) m(A)<\varepsilon / 2 .
$$

Finally,

$$
m\left[T^{n}(E \cap A) \cap(E \cap A)\right]>m\left[T^{n}(E \cap A)\right]-\varepsilon / 8-\varepsilon / 2=m(E \cap A)-5 \varepsilon / 8 .
$$

Combining these estimates, we have

Hence

$$
m(E \cap A) \leqslant m\left[T^{n}(E \cap A) \cap(E \cap A)\right]+\varepsilon .
$$

$$
\left|m\left(T^{n} E \cap E\right)-m(E \cap A)\right|<\varepsilon .
$$

Moreover, since $m(E \cap A)>m(E)-\varepsilon$, we have

$$
m\left(T^{n} E \cap E\right)>m(E)-2 \varepsilon .
$$

We conclude that

$$
\limsup _{n \rightarrow \infty} m\left(T^{n} E \cap E\right) \geqslant m(E)-2 \varepsilon>m^{2}(E),
$$

as desired. 


\section{REFERENCES}

1. N. A. Friedman, Introduction to ergodic theory, Van Nostrand, New York, 1970.

2. P. Shields, Cutting and independent stacking of intervals, Math. Systems Theory 7 (1973), 1-4.

3. Ya. G. Sinai, Weak isomorphism of transformations with invariant measure, Amer. Math. Soc. Transl. (2) 57 (1966), 123-143.

Department of Mathematics, University of Massachusetts, Amherst, Massachusetts 01003

Current address: Department of Mathematics, Union College, Schenectady, New York 12308 